\title{
Optimal Projection Pattern for Active Visual Servoing (AVS)
}

\author{
Shogo Arai $^{1}$, Member, IEEE, Yoshihiro Miyamoto ${ }^{2}$, Akinari Kobayashi ${ }^{2}$, Member, IEEE, \\ and Kazuhiro Kosuge ${ }^{1}$, Fellow, IEEE
}

\begin{abstract}
Visual servo control uses images that are obtained by a camera for robotic control. This study focuses on the problem of positioning a target object using a robotic manipulator with image-based visual servo (IBVS) control. To perform the positioning task, the image-based visual servoing requires visual features that can be extracted from the appearance of the target object. Therefore, a positioning error tends to increase especially for textureless objects, such as industrial parts. Since it is difficult to extract differences in the visual features between current and goal images. To solve these problems, this paper presents a novel visual servoing named "Active Visual Servoing." Active Visual Servoing (AVS) projects patterned light onto the target object using a projector. The design of the projection pattern affects the positioning error. AVS uses an optimal pattern that is theoretically derived and maximizes differences between current and goal images. The experimental results show that the proposed active visual servoing method reduces the positioning error by more than $97 \%$ compared to conventional image-based visual servoing.
\end{abstract}

Index Terms-Kitting, positioning, visual servoing, grasping error.

\section{INTRODUCTION}

$\mathbf{V}$ ISUAL servoing is a widely used method for positioning [1]-[7]. One important application of visual servoing is positioning task in factory automation since the positioning task is required after random bin-picking [8]-[11] to correct grasping error deviated from grasp planning [12] and perform assembling tasks [13]. This study, in particular, considers the problem of positioning an object grasped by a hand attached to a manipulator. The visual servoing is roughly classified into position-based (PBVS) and image-based visual servoing (IBVS) [14], and this paper focuses on IBVS.

IBVS calculates the control input such that the error of the visual feature between the image of the target object at the current time and the goal image converges to zero [15], as shown in Fig. 1. Because IBVS does not need to estimate the pose of the target object from images, the positioning accuracy of IBVS is not influenced by the camera-robot calibration error, the image quantization error, and the modeling error of the camera, all of them are problems of PBVS. However, it is difficult to perform highly accurate positioning of an object with poor visual features, such as a textureless object. Even

\footnotetext{
${ }^{1}$ Shogo Arai and Kazuhiro Kosuge are with Faculty of Graduate School of Engineering, Department of Robotics, Tohoku University, Sendai, Japan \{shogo.arai.d3, kosuge.kazuhiro.e3\}atohoku.ac.jp

${ }^{2}$ Yoshihiro Miyamoto, and Akinari Kobayashi are with the Graduate School of Engineering, Tohoku University, Sendai, Japan \{miyamoto, kobayashi\}@irs.mech.tohoku.ac.jp
}

if the visual features can be extracted, a convergence error (positioning error) tends to remain due to the small deviation of the visual feature between the target and current pose. Further, it is necessary to extract the image features ${ }^{1}$, convert them to visual features for IBVS, and to track them between the goal and current images at each time. This process is not feasible especially for objects with poor image features.

To solve these problems, we propose a novel visual servoing named "Active Visual Servoing" (AVS). AVS uses a projector to irradiate an object with a light pattern and a camera to capture the reflected light. By directly using the luminance values of each pixel in the captured image as the visual feature, visual servoing is performed, as shown in Fig. 2. The proposed method does not require extracting the image features, converting the extracted image features to the visual features, and tracking them.

The positioning accuracy of the proposed active visual servoing mainly depends on the projection pattern. Image deviation of the objects with poor features becomes too small near the goal pose. We theoretically derive the optimal light pattern that maximizes image deviation near the goal poses to reduce the convergence of the positioning error. From this point of view, the active visual servoing proposed in this paper is a completely new attempt to achieve high accuracy positioning, while the existing methods use the structured light which is not optimized for IBVS.

AVS has the following properties:

1) The proposed active visual servoing retains the advantages of IBVS, that is, the positioning accuracy does not depend on the calibration, quantization, and modeling errors of the camera.

2) Since the proposed method uses the luminance values of the image as the visual feature and does not require extraction of the feature from the image, the proposed active visual servoing can easily be applied to the objects with poor image features, such as textureless objects, industrial parts, and so on.

3) The optimal light pattern for AVS does not depend on the object shape under some assumptions.

4) The proposed method has high positioning accuracy compared to the conventional IBVS, which was verified

\footnotetext{
${ }^{1}$ In this paper, the image feature represents the feature directly extracted from the captured image, such as point, edge, SIFT, SURF. On the other hand, the visual feature is computed from the image feature and used for IBVS, such as the image coordinates of the corners, edges, and keypoints for SIFT or SURF
} 


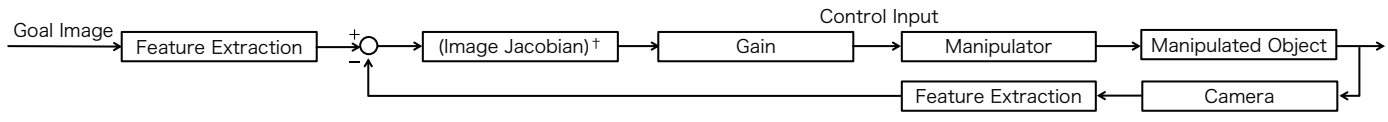

Fig. 1: Block diagram of image-based visual servoing system.

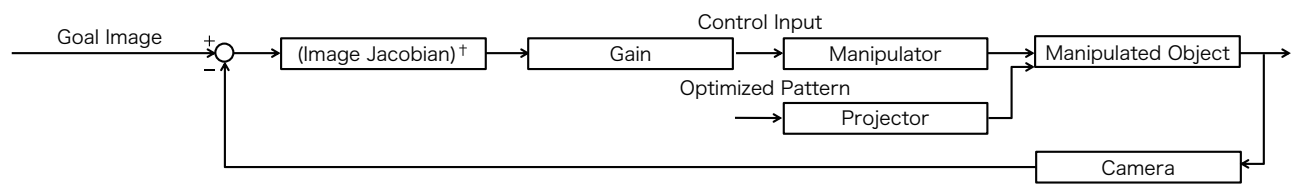

Fig. 2: Block diagram of active visual servoing system proposed in this paper.

through comparative experiments using actual industrial parts.

This paper is organized as follows: Sec. II describes related works. Sec. III proposes active visual servo control and sec. IV derives the optimal light pattern irradiating from the projector for active visual servoing. Sec. V presents the experimental results for the validation of active visual servoing and sec. VI concludes this paper.

This paper uses the following notations. The symbols $\mathbf{0}_{n}$ and $\mathbf{1}_{n}$ represent $n$-dimensional vectors whose all elements are 0 and 1 , respectively.

\section{RELATED WORKS}

In the field of three-dimensional (3D) measurement, a number of methods have been proposed [16]-[19]. Besides, the obtained 3D point cloud or depth image are utilized for various robotic applications involving robot vision, such as bin-picking [11], [20], prediction of reaching motion [21], [22], 3D keypoint detection [23], [24], 3D feature description [25], segmentation [26], [27], and SLAM [28]. An example of the representative three-dimensional measurement method is the light-section method, in which $3 \mathrm{D}$ measurement is performed by projecting a line of light and measuring its reflected light. The light-section method takes some time for the measurement because it is necessary to scan multiple lines of light for measuring the entire surface of the target object. This paper focuses on active stereo methods that do not require line-by-line scanning. Active stereo methods perform three-dimensional measurement by irradiating a target object with a pre-designed light pattern and by measuring the light reflected by the object [29]-[31]. As the representative methods, space coding [32] and phase shift methods [33] have been proposed and various other methods using the camera and projector have been developed [34]-[36]. In these methods, the design of the patterned light is a major factor determining the accuracy and measurement time. Major advantages of the active stereo methods are that they are robust to changes in ambient light and they can perform 3D measurements of an object irrespective of the presence or absence of a surface pattern on the object. Inspired by a concept of the active stereo methods, this paper proposes the active visual servoing that accomplishes the object positioning with higher accuracy than the conventional IBVS.

Image-based visual servoing using a laser have been proposed in [37]-[39]. Pagès et al. have proposed visual servoing with lasers for positioning the camera attached to the tip of the end effector [38]. This method involves the use of four lasers to irradiate a planar target, and visual feature is calculated by using parts of images surrounding the irradiated points. The authors have shown that this method can achieve robust positioning of the camera and decouple control of rotation around the optical axis of the camera from the other two axes. However, because of the complexity of the interaction matrix, this method does not guarantee global stability. They have subsequently proposed a solution to this problem by using a simple visual feature composed of only the image coordinates of irradiated points [39]. This approach has shown global stability under ideal circumstances, i.e., global stability is guaranteed when a placement error between the camera and laser is zero. Experimental results have shown that these visual servoing methods performed positioning of the camera with respect to a planar target object.

In addition to the above-mentioned work, a visual servo control method that projects a pattern onto an object by using a projector has been proposed [40], [41]. This method entails the generation of dot patterns with three colors, red, green, blue, based on the M-array and the projection of these patterns onto the target object. The method functions by matching the projected dots between the current and goal images; then, by using the matching results, the method performs positioning based on IBVS. The projected patterns based on the Marray facilitate identification of the correspondence dots among images captured by cameras with different viewpoints. The method requires processes of segmentation to extract dots, computing the position of the centroid of the dots, finding the dots nearest to each of the dots, and checking the graph consistency. The effectiveness of the method for objects with complicated shapes has not been verified, although verification experiments with planar and elliptic cylinders have been conducted.

The advantages of the method proposed in this paper are as follows:

1) Image processing for the proposed active visual servoing method is a high speed, since the luminance value of each pixel is directly used as the visual feature;

2) Existing structured light is not used. Instead, we analytically derive the optimal patterned light to be irradiated by the projector such that the cost function is minimized;

3) The experimental results confirm that the proposed method can perform positioning of complex-shaped objects with higher accuracy than the conventional visual 
servoing.

\section{Principle of IMAGe-BASEd VISUAL SERVo CONTROL}

This section describes the basic principles of IBVS. The purpose here is to position a target object grasped by a robotic hand equipped with a manipulator. A camera fixed to the world system captures the target object. The goal image is obtained by capturing the target object located at the goal pose

IBVS provides the control input to the manipulator by using the visual features computed from the captured image, as shown in Fig. 1. The visual feature is a multidimensional vector and can be computed based on the image feature, such as the edges, counters, corners, SIFT [44], SURF [45], AKAZE [46], etc., without using the relative pose of the camera to the manipulator and the intrinsic parameter of the camera. The purpose of IBVS is to minimize the objective function defined by

$$
e(t)=f(I(t))-f\left(I^{*}\right),
$$

where $I$ and $I^{*}$ represent vectors whose elements are intensities of each pixel in the current and goal images, respectively. The symbol $f(I)$ denotes the mapping from image $I$ to the visual feature. The simplest control law to achieve this purpose is represented by

$$
\dot{\theta}_{\mathrm{d}}=-\lambda J^{\dagger}\left(f(I(t))-f\left(I^{*}\right)\right),
$$

where $\dot{\theta}_{\mathrm{d}} \in \mathbb{R}^{n}$ is the control input of the joint angular velocity for the manipulator, $n$ is the degree of freedom (DOF) of the manipulator, $\lambda \in \mathbb{R}$ is the gain, and $J^{\dagger}$ is the pseudo-inverse matrix of the image Jacobian.

\section{ACtive Visual SERVo CONTROL SCHEME AND OPTIMAL PROJECTION PATTERN}

This section proposes the active visual servo control scheme. The proposed method uses a projector to project optimized patterned light onto the target object and a camera to capture an image of the reflected light, as shown in Fig. 3. Then the control input is computed by multiplying a pseudo-inverse matrix of image Jacobian by an image difference between current and goal images, as shown in Fig. 2. This section presents the control law for active visual servoing and derives the optimal projection pattern that affects the positioning accuracy.

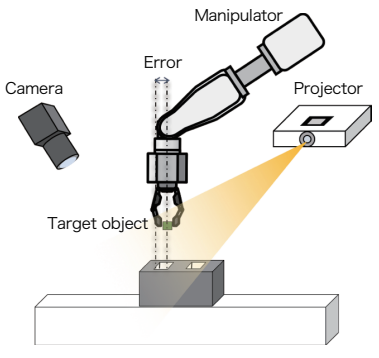

Fig. 3: System configuration for proposed active visual servoing.

\section{A. Control law of Active Visual Servoing}

This subsection explains the control law of the active visual servoing. Active visual servo control directly utilizes the intensity of each pixel in the captured image as the visual feature, which is inspired by direct visual servoing [47]. The control law of AVS is shown below:

$$
\dot{\theta}_{\mathrm{d}}=-\lambda J^{\dagger}\left(I(t)-I^{*}\right),
$$

The control law (3) can be obtained by replacing the visual feature $f$ with the image $I$ in (2). The control law (3) does not require computation for the extraction of image features and thus can be computed faster than one by (2).

The pseudo-inverse matrix of the image Jacobian $J^{\dagger}$ in (3) represents a mapping from the error between the current and goal images to the joint angular velocity and depends on the joint angles of the manipulator. In this paper, the image Jacobian is assumed to be the constant and approximated by the one at the goal pose.

\section{B. Optimal projection pattern for Active Visual Servoing}

This subsection derives the optimal projection pattern for AVS. Let us consider a three-dimensional space as shown in Fig. 4. The manipulator, projector, and camera are fixed in the space and the coordinate system $\Sigma_{\mathrm{c}}$ and $\Sigma_{\mathrm{p}}$ are set such that the origins coincide with their optical centers and the $z$-axes are parallel to the optical axis of the camera and projector, respectively. In the following discussions, poses are represented based on the camera coordinate system unless otherwise noted.

Now we assume the simplest pinhole camera model, that is, a 3D point located at $(x, y, z)$ is projected at

$$
\begin{aligned}
X_{\mathrm{c}} & =\frac{f_{\mathrm{cx}}}{z} x, \\
Y_{\mathrm{c}} & =\frac{f_{\mathrm{cy}}}{z} y
\end{aligned}
$$

in the image plane of the camera, where $f_{\mathrm{cx}}$ and $f_{\mathrm{cy}}$ are the focal lengths.

The projector is set at $\xi_{\mathrm{p}}:=\left[x_{\mathrm{p}}, y_{\mathrm{p}}, z_{\mathrm{p}}, \theta_{\mathrm{p}}, \phi_{\mathrm{p}}, \psi_{\mathrm{p}}\right]^{\top}$, where $\left(x_{\mathrm{p}}, y_{\mathrm{p}}, z_{\mathrm{p}}\right)$ is the position of the projector and $\left(\theta_{\mathrm{p}}, \phi_{\mathrm{p}}, \psi_{\mathrm{p}}\right)$ is the attitude of the projector. In other words, the projector coordinate system is set at $\xi_{\mathrm{p}}$ in the camera coordinate system. It is assumed that the optical model of the projector also follows the pinhole camera model.

We denote the poses of the target object at the current time and the goal pose by $\xi_{0}:=\left[x_{0}, y_{0}, z_{0}, \theta_{0}, \phi_{0}, \psi_{0}\right]^{\top}$ and $\xi_{\mathrm{o}}^{*}:=\left[x_{\mathrm{o}}^{*}, y_{\mathrm{o}}^{*}, z_{\mathrm{o}}^{*}, \theta_{\mathrm{o}}^{*}, \boldsymbol{\phi}_{\mathrm{o}}^{*}, \psi_{\mathrm{o}}^{*}\right]^{\top}$, respectively. The surface shape of the target object at the pose $[0,0,0,0,0,0]^{\top}$ is represented by $[\alpha(\tau), \beta(\tau), \gamma(\tau)]^{\top}$, where $\tau \in \mathbb{R}^{2}, \mathbf{0}_{2} \leq \tau \leq \mathbf{1}_{2}$ is a parameter used for representing a curved surface. By using this notation, the surface of the target object at pose $\xi_{0}$ can be represented by

$$
s\left(\xi_{\mathrm{o}}, \tau\right):=R\left(\theta_{\mathrm{o}}, \phi_{\mathrm{o}}, \psi_{\mathrm{o}}\right)\left[\begin{array}{l}
\alpha(\tau) \\
\beta(\tau) \\
\gamma(\tau)
\end{array}\right]+\left[\begin{array}{l}
x_{\mathrm{o}} \\
y_{\mathrm{o}} \\
z_{\mathrm{o}}
\end{array}\right],
$$

where $R\left(\theta_{\mathrm{o}}, \phi_{\mathrm{o}}, \psi_{\mathrm{o}}\right)$ represents a rotation matrix. 


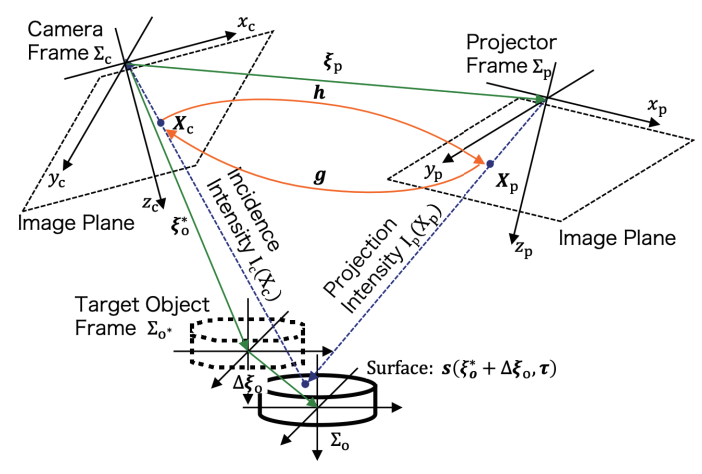

Fig. 4: Arrangement of a camera, projector, and target object in a problem to the derive optimal projection pattern.

The light projected from $\boldsymbol{X}_{p}:=\left[X_{\mathrm{p}}, Y_{\mathrm{p}}\right]^{\top} \in \mathbb{R}^{2}$ in the image plane of the projector is reflected at $s\left(\xi_{\mathrm{o}}^{*}, \tau\right)$ on the surface of the object with pose $\xi_{\mathrm{o}^{*}}$ and then reaches $\boldsymbol{X}_{\mathrm{c}}:=\left[X_{\mathrm{c}}, Y_{\mathrm{c}}\right]^{\top} \in \mathbb{R}^{2}$ in the image plane of the camera. By using a mapping $g$ from the image plane of the projector to that of the camera, this relationship can be denoted by

$$
\boldsymbol{X}_{\mathrm{c}}=g\left(\boldsymbol{X}_{\mathrm{p}}, s\left(\xi_{\mathrm{o}}^{*}, \tau\right), \xi_{\mathrm{p}}\right) .
$$

Next, we consider the pattern of the light projected by the projector. The function for the projected light pattern is defined by $I_{\mathrm{p}}\left(\boldsymbol{X}_{\mathrm{p}}\right)$, where $I_{\mathrm{p}}\left(\boldsymbol{X}_{\mathrm{p}}\right)$ is the intensity of the projection at $\boldsymbol{X}_{\mathrm{p}}$ in the image of the projector.

To simplify the discussion, it is assumed that the intensity of the light incident on a certain pixel in the image plane of the camera is equal to the one from the corresponding pixel in the image plane of the projector. By considering that the incident ray on $\boldsymbol{X}_{\mathrm{c}}$ in the image plane of the camera is projected from $\boldsymbol{X}_{p}$ in the image plane of the projector, the relationship for the intensity

$$
I_{\mathrm{c}}\left(\boldsymbol{X}_{c}\right)=I_{\mathrm{p}}\left(\boldsymbol{X}_{p}\right)
$$

holds. By using the inverse function of $g$, which is denoted by $h$, eq. (8) can be rewritten by

$$
I_{\mathrm{c}}\left(\boldsymbol{X}_{\mathrm{c}}\right)=I_{\mathrm{p}}\left(h\left(\boldsymbol{X}_{\mathrm{c}}, s\left(\xi_{\mathrm{o}}^{*}, \tau\right), \xi_{\mathrm{p}}\right)\right) .
$$

As shown in the above equation, $I_{\mathrm{c}}$ actually relies on the pose and the shape of the object. For later discussion, $I_{\mathrm{c}}\left(\boldsymbol{X}_{\mathrm{c}}\right)$ is denoted by $I_{\mathrm{c}}\left(\boldsymbol{X}_{\mathrm{c}}, s, \xi_{\mathrm{o}}^{*}\right)$.

When the target object moves by $\Delta \xi_{\mathrm{o}}$ from the target pose $\xi_{\mathrm{o}}^{*}$, the incident ray on the same pixel $\boldsymbol{X}_{\mathrm{c}}$ is considered to be projected from

$$
\boldsymbol{X}_{\mathrm{p}}+\Delta \boldsymbol{X}_{\mathrm{p}}=h\left(\boldsymbol{X}_{\mathrm{c}}, s\left(\xi_{\mathrm{o}}^{*}+\Delta \xi_{\mathrm{o}}, \tau\right), \xi_{\mathrm{p}}\right)
$$

in the image plane of the projector. Therefore, the intensity observed at $\boldsymbol{X}_{\mathrm{c}}$ in the image plane of the camera is

$$
I_{\mathrm{c}}\left(\boldsymbol{X}_{\mathrm{c}}, s, \xi_{\mathrm{o}}^{*}+\Delta \xi_{\mathrm{o}}\right)=I_{\mathrm{p}}\left(h\left(\boldsymbol{X}_{\mathrm{c}}, s\left(\xi_{\mathrm{o}}^{*}+\Delta \xi_{\mathrm{o}}, \tau\right), \xi_{\mathrm{p}}\right)\right) .
$$

As shown in eq. (3), active visual servo control uses the intensities of the image as the visual features. To decrease the positioning error and the time required for positioning, we derive the optimal projection pattern that maximizes the image error in (3), that is, the optimal projection pattern is denoted by

$$
I_{\mathrm{p}}^{*}=\arg \max _{I_{\mathrm{p}}} c\left(I_{\mathrm{p}}\right),
$$

where $c\left(I_{\mathrm{p}}\right)=\left|\gamma\left(I_{\mathrm{p}}\right)\right|^{2}$. The symbol $\gamma\left(I_{\mathrm{p}}\right)$ represents the error of luminance captured by the camera between the goal and current poses of the object, which is defined by

$$
\gamma\left(I_{\mathrm{p}}\right)=I_{\mathrm{c}}\left(\boldsymbol{X}_{\mathrm{c}}, s, \xi_{\mathrm{o}}\right)-I_{\mathrm{c}}\left(\boldsymbol{X}_{\mathrm{c}}, s, \xi_{\mathrm{o}}^{*}\right) .
$$

By using the relationship given by (9), the Taylor expansion of $\gamma\left(I_{\mathrm{p}}\right)$ for in the vicinity of the goal pose is derived as follows:

$$
\begin{aligned}
\gamma\left(I_{\mathrm{p}}\right)= & I_{\mathrm{p}}\left(h\left(\boldsymbol{X}_{\mathrm{c}}, s\left(\xi_{\mathrm{o}}^{*}+\Delta \xi_{\mathrm{o}}\right), \xi_{\mathrm{p}}\right)\right)-I_{\mathrm{p}}\left(h\left(\boldsymbol{X}_{\mathrm{c}}, s\left(\xi_{\mathrm{o}}^{*}\right), \xi_{\mathrm{p}}\right)\right) \\
= & I_{\mathrm{p}}\left(h\left(\boldsymbol{X}_{\mathrm{c}}, s\left(\xi_{\mathrm{o}}^{*}\right), \xi_{\mathrm{p}}\right)+\left.\left.\frac{\partial h}{\partial s^{\top}}\right|_{s=s\left(\xi_{\mathrm{o}}^{*}\right)} \frac{\partial s}{\partial \xi_{\mathrm{o}}^{\top}}\right|_{\xi_{\mathrm{o}}=\xi_{\mathrm{o}}^{*}} \Delta \xi_{\mathrm{o}}\right) \\
& -I_{\mathrm{p}}\left(h\left(\boldsymbol{X}_{\mathrm{c}}, s\left(\xi_{\mathrm{o}}^{*}\right), \xi_{\mathrm{p}}\right)\right)+O\left(\Delta \xi_{\mathrm{o}}^{2}\right) \\
= & \left.\frac{\partial I_{\mathrm{p}}}{\partial h^{\top}}\right|_{h=h\left(\boldsymbol{X}_{\mathrm{c}}, s\left(\xi_{\mathrm{o}}^{*}\right), \xi_{\mathrm{p}}\right)} \underbrace{\left.\frac{\partial h}{\partial s^{\top}}\right|_{s=s\left(\xi_{\mathrm{o}}^{*}\right)}}_{\mathrm{A}} \underbrace{\left.\frac{\partial s}{\partial \xi_{\mathrm{o}}^{\top}}\right|_{\xi_{\mathrm{o}}=\xi_{\mathrm{o}}^{*}} \Delta \xi_{\mathrm{o}}}_{\mathrm{B}} \\
& +O\left(\Delta \xi_{\mathrm{o}}^{2}\right),
\end{aligned}
$$

where $O\left(\Delta \xi_{\mathrm{o}}^{2}\right)$ is the secondary or higher remainder term of the Taylor expansion and $s(\xi, \tau)$ is denoted by $s(\xi)$ to simplify notations. Neglecting $O\left(\Delta \xi_{\mathrm{o}}^{2}\right)$ and substituting (15) to $c(I)$ derives

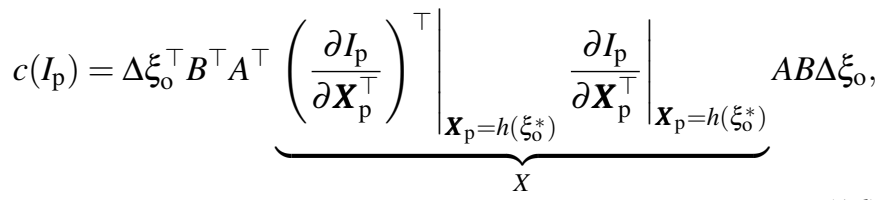

where we use the relation $\boldsymbol{X}_{\mathrm{p}}=h\left(\xi_{\mathrm{o}}^{*}\right)$ and $h\left(\boldsymbol{X}_{\mathrm{c}}, s\left(\xi_{\mathrm{o}}^{*}, \tau\right), \xi_{\mathrm{p}}\right)$ is denoted by $h\left(\xi_{0}^{*}\right)$ for simplifying notation. In (15) and (16), the matrices $A, B$, and $X$ depend on the shape of the target object, the poses of the object, and the projected pattern, respectively. Because we can control only matrix $X$ in $c\left(I_{\mathrm{p}}\right)$ by changing the projection pattern, For maximizing $c\left(I_{\mathrm{p}}\right)$ for arbitrary vector $\varepsilon:=A B \Delta \xi_{\mathrm{o}}$, we needs to maximize the eigenvalues of $X$ that are given by

$$
\lambda(X)=\left\{0, \quad\left(\frac{\partial I_{\mathrm{p}}}{\partial X_{\mathrm{p}}}\right)^{2}+\left(\frac{\partial I_{\mathrm{p}}}{\partial Y_{\mathrm{p}}}\right)^{2}\right\} .
$$

As shown in eq. (17), the first eigenvalue is equal to zero ${ }^{2}$. Hence, we consider the projected pattern which maximizes a second eigenvalue:

$$
I_{\mathrm{p}}^{*}=\arg \max _{I_{\mathrm{p}}}\left\{\left(\frac{\partial I_{\mathrm{p}}}{\partial X_{\mathrm{p}}}\right)^{2}+\left(\frac{\partial I_{\mathrm{p}}}{\partial Y_{\mathrm{p}}}\right)^{2}\right\}
$$

Eq. (18) means that the optimized projection pattern maximizes the square of first derivative of intensity with the image coordinates $X_{\mathrm{p}}$ and $Y_{\mathrm{p}}$ in the image plane of the projector.

${ }^{2}$ We can interpret this fact as follows: regardless of projected patterns, no image difference occurs near the goal pose in a special case where a target object has a planar surface and the surface of the object is perpendicular to the optical axis of the camera in the goal pose. 
In the above discussion, we assumed the resolutions of both the camera and projector to be infinite. In practice, the resolutions are finite and the image planes of both the camera and projector consist of a finite number of pixels. Considering this point, we denote the projection pattern by $I_{\mathrm{d}}\left(X_{\mathrm{pd}}, Y_{\mathrm{pd}}\right)$, $X_{\mathrm{pd}} \in\left\{1,2, \cdots, w_{\mathrm{p}}\right\}, Y_{\mathrm{pd}} \in\left\{1,2, \cdots, h_{\mathrm{p}}\right\}$ where $w_{\mathrm{p}}$ and $h_{\mathrm{p}}$ are the resolutions of the projector. Two terms in right hand side of eq. (18) can be approximated by forward difference as follows:

$$
\begin{aligned}
& \left(\frac{\partial I_{\mathrm{p}}}{\partial X_{\mathrm{p}}}\right)^{2} \approx\left(I\left(X_{\mathrm{pd}}^{*}+1\right)-I\left(X_{\mathrm{pd}}^{*}\right)\right)^{2}=: \bar{I}_{X}, \\
& \left(\frac{\partial I_{\mathrm{p}}}{\partial Y_{\mathrm{p}}}\right)^{2} \approx\left(I\left(Y_{\mathrm{pd}}^{*}+1\right)-I\left(Y_{\mathrm{pd}}^{*}\right)\right)^{2}=: \bar{I}_{Y},
\end{aligned}
$$

where

$$
\begin{gathered}
X_{\mathrm{pd}}^{*}=f_{\mathrm{sX}}\left(h_{X}\left(\boldsymbol{X}_{\mathrm{c}}, s\left(\xi_{\mathrm{o}}^{*}, \tau\right), \xi_{\mathrm{p}}\right)\right), \\
Y_{\mathrm{pd}}^{*}=f_{\mathrm{s} Y}\left(h_{Y}\left(\boldsymbol{X}_{\mathrm{c}}, s\left(\xi_{\mathrm{o}}^{*}, \tau\right), \xi_{\mathrm{p}}\right)\right) .
\end{gathered}
$$

Here $h_{X}$ and $h_{Y}$ are first and second element of vector function $h$, and $f_{\mathrm{sX}}, f_{\mathrm{sY}}$ are step functions defined by

$$
\begin{aligned}
& f_{\mathrm{sX}}(\zeta)=\sum_{i=1}^{w_{\mathrm{p}}} i \chi_{i}(\zeta) \\
& f_{\mathrm{sY}}(\zeta)=\sum_{i=1}^{h_{\mathrm{p}}} i \chi_{i}(\zeta)
\end{aligned}
$$

Here $\chi_{i}$ is an indicator function represented by

$$
\chi_{i}(\zeta)= \begin{cases}1, & (\zeta \in[i, i+1)), \\ 0, & (\zeta \notin[i, i+1)) .\end{cases}
$$

Now we can obtain the maxima of $\bar{I}_{X}$ in eq. (19) and $\bar{I}_{Y}$ in eq. (20) by

$$
\begin{aligned}
& \bar{I}_{X}=\left(I_{\mathrm{p}, \text { max }}-I_{\mathrm{p}, \text { min }}\right)^{2}, \\
& \bar{I}_{Y}=\left(I_{\mathrm{p}, \text { max }}-I_{\mathrm{p}, \text { min }}\right)^{2},
\end{aligned}
$$

where $I_{\mathrm{p}, \max }$ and $I_{\mathrm{p}, \min }$ are the maximum and minimum intensities of the projection, respectively. It is obvious that a checkered pattern as shown in Fig. 5 can satisfies conditions represented by eqs. (26) and (27) simultaneously. Hence AVS adopts the striped patterns and we validate the positioning performance of AVS with the striped patterns in the following section.

\section{EXPERIMENT}

This section validates the performance of the proposed AVS and compares its performance to the conventional IBVS.

Fig. 6 shows an experimental system used for validation. The validation experiment involves a positioning task for target objects. The target objects are made of plastic material and have complex shapes, as shown in Fig. 7. We computed the image Jacobian by using numerical differentiation. The projector, an EB-W420 (EPSON) with a resolution of $1280 \times 800$ (pixel), was set up at a distance of $(-0.5[\mathrm{~m}], 0[\mathrm{~m}], 0.5[\mathrm{~m}])$ from the target position. The camera, IDP-Express R2000 (Photron), which was located at a distance of $0.1 \mathrm{~m}$ below the projector, was used to capture images sized $512 \times 512$ (pixel) at a frequency of $50(\mathrm{~Hz})$. The captured images were

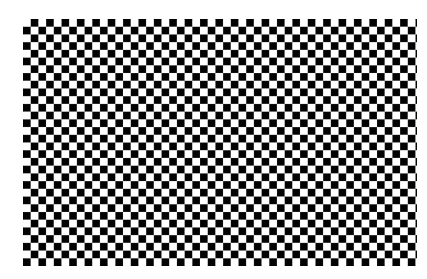

Fig. 5: Checkered pattern.

sent to a PC with an Intel Core i7-4720HQ CPU and 16 GB of DDR3 SDRAM, and processed for control. OpenCV library [48] was used to process the captured images. The manipulator was VS068 from Denso Wave Inc. with six DOFs. A parallel gripper was attached to the manipulator. To measure the positioning error, a laser displacement meter LJ-V7300 from Keyence Corp. was set up at the position shown in Fig. 6 . The direction of the laser irradiation was parallel to the $x$-axis.

The purpose of this experiment was to perform positioning of the target objects shown in Fig. 7. The goal pose of target object A was set as indicated in Fig. 6. The initial position and posture were set to be $(5[\mathrm{~cm}], 5[\mathrm{~cm}], 5[\mathrm{~cm}])$ and (10deg., 10deg., 10deg.) away from the target position and posture, respectively. Here the posture is represented by XYZ Euler angles. In addition, the grasping error was set to be (10deg., 10deg., 10deg.) away from the ideal posture ${ }^{3}$.

The control for the conventional and active visual servo is given by (3). The gain $\lambda$ is set to be 2.0 .

The projector projected the checkered pattern, which is the optimal projection pattern as shown in sec. IV.

In the experimental system, the robot controller requires the control input in a cycle of 1 [ms]. Because the frame rate of the camera is 50 (fps), we need to interpolate the control input. The control input at time $t$ is computed by

$$
\bar{u}(t)=u(n T)+\frac{u(n T)-u((n-1) T)}{T} \bar{t},
$$

where $t=n T+\bar{t}, \bar{t} \in\{0,1, \cdots, T-1\}, n \in\{0,1, \cdots\}$, and $T$ is the sampling period of the camera $(=20[\mathrm{~ms}])$.

The system terminates when sum of squared differences between the current and goal images is less than the specified threshold.

Fig. 8 depicts the time-series images of one representative AVS sequence for target object A. The goal image of the target object was overlaid on the images. It can be seen that the images at each time are changing to coincide with the goal image.

Fig. 9 shows the time series data of joint angular velocity of the manipulator for the proposed active visual servoing, which are corresponding to Fig. 8 .

The sum of squared differences between goal and captured image at each time is presented in Fig. 10. In the figure, the image error is normalized by the error that exists at the initial time. The image errors in both the conventional and proposed AVS converge at approximately $1.5[\mathrm{~s}]$. The steadystate image error of AVS is approximately $12 \%$ smaller than

\footnotetext{
${ }^{3}$ Assuming the object is grasped by the hand at the ideal posture, we computed the image Jacobian by numerical differentiation
} 


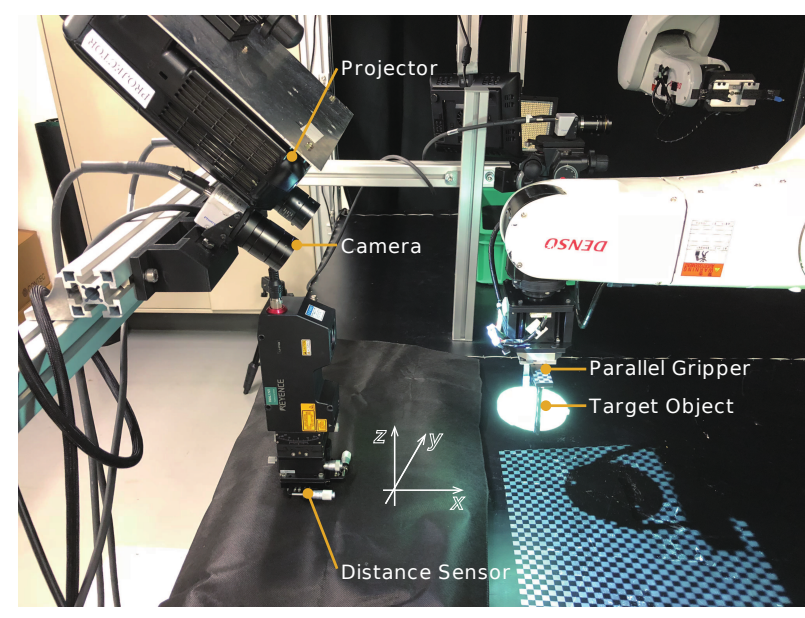

Fig. 6: Experimental environment.
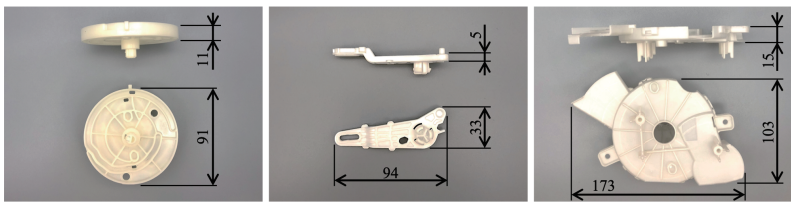

Fig. 7: Target objects. Left: target object A. Middle: target object B. Right: target object C.

that of the conventional IBVS. In addition, whereas the image error for the conventional IBVS increases from $t=1.0[\mathrm{~s}]$ to $t=2.3[\mathrm{~s}]$, that of active visual servo control decreases almost monotonically. Here, note that Fig. 10 shows only the image error but does not show the positioning error in 3D space.

To evaluate the positioning error in 3D space, the laser displacement sensor was used. The positioning errors are measured 10 times for each target object in both the AVS and the conventional IBVS; the results are plotted in Fig. 11. Fig. 11 indicates that AVS decreases the positioning errors by

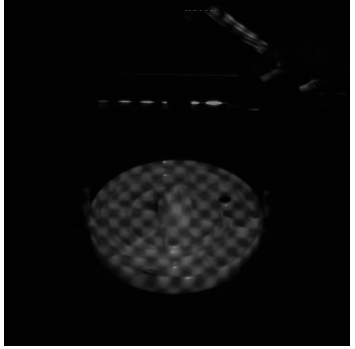

$0.0[\mathrm{~s}]$

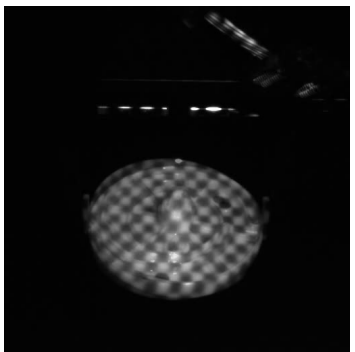

$0.8[\mathrm{~s}]$

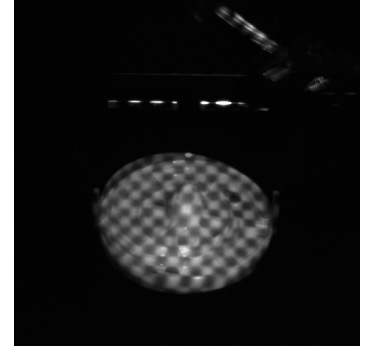

$0.4[\mathrm{~s}]$

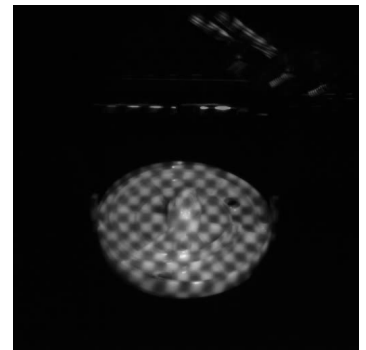

$1.2[\mathrm{~s}]$
Fig. 8: Time series images captured from the camera used for active visual servoing for target object A. Goal image of the target object is overlaid on each image. The optimal pattern is irradiated from the projector. The images show how the goal image and the current image match.

more than $97 \%$ compared to the conventional IBVS.

\section{CONCLUSION}

This paper proposes the active visual servo control (AVS) to perform the positioning of an object, especially a textureless object, with high accuracy. The proposed method uses a projector to project a light pattern onto the target object to increase the image error between the current and goal images. In this method, the light pattern used for irradiation

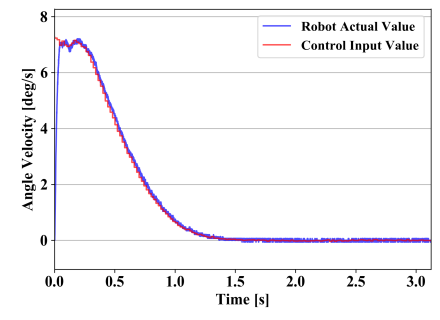

1st joint.

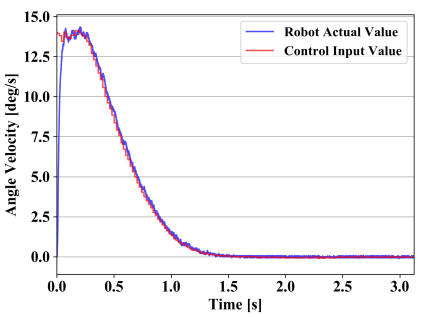

4th joint.

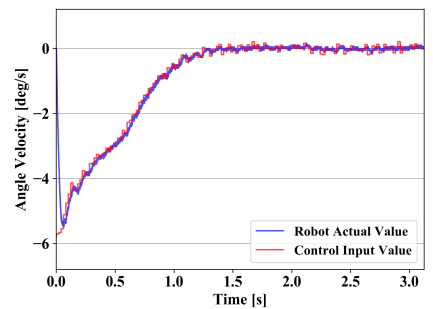

2nd joint.

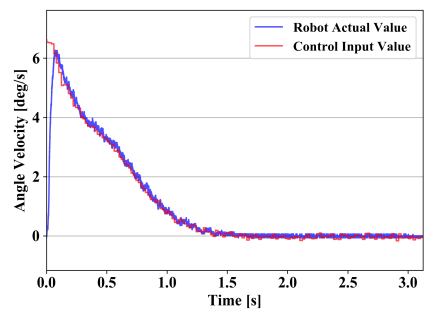

5th joint.

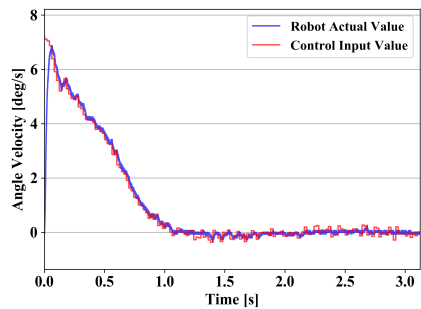

3rd joint.

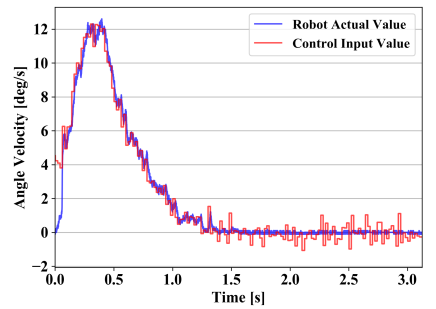

6th joint.

Fig. 9: Time series data of joint angular velocity of the manipulator for the proposed active visual servoing. 


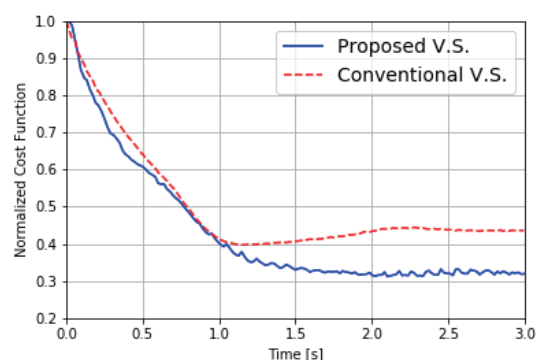

Fig. 10: Time series data of the normalized cost function of IBVS.

has a great influence on the positioning accuracy. Thus, this study derives the optimal pattern that maximizes the image error. The experimental results show that the proposed method achieves higher positioning accuracy and provides much less positioning error that is more than $97 \%$ smaller than that of the conventional IBVS.

\section{REFERENCES}

[1] W. J Wilson, CC W. Hulls, and G. S Bell, "Relative end-effector control using Cartesian position based visual servoing," IEEE Trans. Robot. Auto., vol. 5, pp. 684-696, 1996.

[2] R. Vidal, O. Shakernia, and S. Sastry, "Formation control of nonholonomic mobile robots with omnidirectional visual servoing and motion segmentation," Proc. IEEE Int. Conf. Robot. and Auto., pp. 584-589, 2003.

[3] F. Tokuda, S. Arai, K. Kosuge, "Neural Network based Visual Servoing for Eye-to-Hand Manipulator," TechRxiv, https://doi.org/10.36227/techrxiv.13154075.v1, 2020.

[4] F. Tokuda, S. Arai, and K. Kosuge, "Object Positioning by Visual Servoing Based on Deep Learning," Transactions of the Society of Instrument and Control Engineers, Vol. 55, No. 11, pp. 717-725, 2019.

[5] R. Ginhoux, JA Gangloff, MF De Mathelin, L. Soler, M. M. A. Sanchez, and J. Marescaux, "Beating heart tracking in robotic surgery using 500 $\mathrm{Hz}$ visual servoing, model predictive control and an adaptive observer," Proc. IEEE Int. Conf. Robot. and Auto., pp.274-279, 2004.

[6] N. Guenard, T. Hamel, and R. Mahony, "A practical visual servo control for an unmanned aerial vehicle," IEEE Trans. Robot., vol. 2, pp.331340,2008

[7] C. Kingkan, S. Ito, S. Arai, T. Nammoto, and K. Hashimoto, "Modelbased virtual visual servoing with point cloud data," IEEE/RSJ Int. Conf. Intelligent Robots and Systems, pp. 5549-5555, 2016.

[8] M. Fujita, Y. Domae, A. Noda, G. A. Garcia Ricardez, T. Nagatani, A. Zeng, S. Song, A. Rodriguez, A. Causo, I. M. Chen, and T. Ogasawara, "What are the important technologies for bin picking? Technology analysis of robots in competitions based on a set of performance metrics," Advanced Robotics, DOI:10.1080/01691864.2019.1698463, 2019.

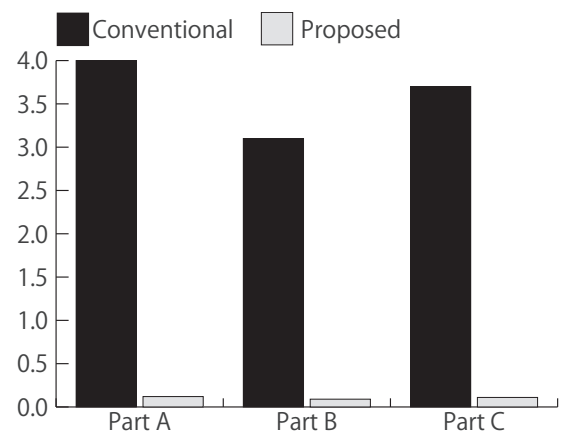

Fig. 11: Positioning errors measured by a distance sensor.
[9] D. Liu, S. Arai, J. Miao, J. Kinugawa, Z. Wang, and K. Kosuge, "Point Pair Feature-Based Pose Estimation with Multiple Edge Appearance Models (PPF-MEAM) for Robotic Bin Picking," Sensors, vol. 18, no. 8, DOI:10.3390/s18082719, 2018.

[10] D. Liu, S. Arai, Y. Xu, F. Tokuda and K. Kosuge, "6D Pose Estimation of Occlusion-Free Objects for Robotic Bin-Picking Using PPF-MEAM With 2D Images (Occlusion-Free PPF-MEAM)," IEEE Access, vol. 9, pp. 50857-50871, 2021.

[11] H. Kuo, H. Su, S. Lai and C. Wu, "3D object detection and pose estimation from depth image for robotic bin picking," IEEE International Conference on Automation Science and Engineering, pp. 1264-1269, 2014.

[12] T. Yamawaki and M. Yashima, "Grasp Planning based on Dynamics Shaping," Proc. IEEE/ASME Int'l. Conf. on Advanced Intelligent Mechatronics, pp. 617-622, 2011.

[13] Y. Yokokohji, Y. Kawai, M. Shibata, Y. Aiyama, S. Kotosaka, W. Uemura, A. Noda, H. Dobashi, T. Sakaguchi, and K. Yokoi, "Assembly Challenge: a robot competition of the Industrial Robotics Category, World Robot Summit - summary of the pre-competition in 2018," Advanced Robotics, vol. 33, no. 17, pp. 876-899, 2019.

[14] F. Chaumette and S. Hutchinson, "Visual servo control. I. Basic approaches," IEEE Robot. \& Auto. Magazine, vol. 13, no. 4, pp. 82-90, 2006.

[15] K. Hashimoto and T. Kimoto and T. Ebine and H. Kimura, "Manipulator control with image-based visual servo," Proc. Int. Conf. Robot. and Auto., pp. 2267-2271, 1991.

[16] M. Mimou, T. Kanade, and T. Sakai, "A method of time-coded parallel planes of light for depth measurement," IEICE Trans., vol. 64, no. 8, pp. 521-528, 1981.

[17] T. Chen, H. P. Seidel, and H. P. A. Lensch, "Modulated phase-shifting for 3D scanning," Proc. Conf. Computer Vision and Pattern Recog., pp. $1-8,2008$.

[18] S Arai, Y Iwatani, K Hashimoto, "Fast sensor scheduling for spatially distributed sensors, "IEEE transactions on automatic control, vol. 56, no. 8, pp. 1900-1905, 2011.

[19] S Arai, Y Iwatani, K Hashimoto, "Fast sensor scheduling with communication costs for sensor networks, "Proceedings of the American Control Conference, pp. 295-300, 2010.

[20] D. Liu, S. Arai, Z. Feng, J. Miao,Y. Xu, J. Kinugawa, and K. Kosuge, "2D Object Localization Based Point Pair Feature for Pose Estimation," IEEE Int'l. Conf. on Robotics and Biomimetics, pp. 1119-1124, 2018.

[21] J. Mainprice, R. Hayne and D. Berenson, "Predicting human reaching motion in collaborative tasks using Inverse Optimal Control and iterative re-planning," 2015 IEEE International Conference on Robotics and Automation (ICRA), Seattle, WA, 2015, pp. 885-892.

[22] S. Arai, A. L. Pettersson and K. Hashimoto, "Fast Prediction of a Worker's Reaching Motion without a Skeleton Model (F-PREMO)," IEEE Access, vol. 8, no. 1, pp. 90340-90350, 2020.

[23] S. Azimi, B. Lall and T. K. Gandhi, "Performance Evalution of 3D Keypoint Detectors and Descriptors for Plants Health Classification," International Conference on Machine Vision Applications (MVA), pp. 1-6, doi: 10.23919/MVA.2019.8758002, 2019.

[24] S. Arai, N. Fukuchi and K. Hashimoto, "FAst Detection Algorithm for 3D Keypoints (FADA-3K)," IEEE Access, 10.1109/ACCESS.2020.3025534, 2020.

[25] D. Liu, S. Arai, F. Tokuda, Y. Xu, J. Kinugawa and K. Kosuge, "DeepLearning based Robust Edge Detection for Point Pair Feature-based Pose Estimation with Multiple Edge Appearance Models," IEEE International Conference on Robotics and Biomimetics, pp. 2920-2925, 2019.

[26] Y. Guo, H. Wang, Q. Hu, H. Liu, L. Liu, and M. Bennamoun, "Deep Learning for 3D Point Clouds: A Survey," IEEE Transactions on Pattern Analysis and Machine Intelligence, doi: 10.1109/TPAMI.2020.3005434.

[27] Y. Xu, S. Arai, F. Tokuda, and K. Kosuge, "A Convolutional Neural Network for Point Cloud Instance Segmentation in Cluttered Scene Trained by Synthetic Data without Color," IEEE Access, Vol. 8, pp. 70262-70269, DOI: 10.1109/ACCESS.2020.2978506, 2020.

[28] S. Lee, C. Hsu, M. Lee, Y. Fu, F. Atas and A. Tsai, "Fast Point Cloud Feature Extraction for Real-time SLAM," International Automatic Control Conference (CACS), pp. 1-6, doi: 10.1109/CACS47674.2019.9024355, 2019.

[29] J. L Posdamer, and MD Altschuler, "Surface measurement by spaceencoded projected beam systems," Computer graphics and image processing, vol. 18, no. 1, pp. 1-17, 1982.

[30] J. Salvi, S. Fernandez, T. Pribanic, and X. Llado, "A state of the art in structured light patterns for surface profilometry," Pattern Recog., vol. 43, no. 8, pp.2666-2680, 2010. 
[31] M. Gupta, A. Agrawal, A. Veeraraghavan, and S. G. Narasimhan, "Structured light 3D scanning in the presence of global illumination," Proc. Conf. Computer Vision and Pattern Recog., pp. 713-720, 2011.

[32] J. L. Posdamer and M. D. Altschuler, "Surface measurement by space encoded projected beam system," Computer Graph. and Image Processing, vol. 18 , no. 1 , pp. 1-17, 1982.

[33] T. Chen, H. PA Lensch, C. Fuchs, and H. P. Seidel, "Polarization and phase-shifting for 3D scanning of translucent objects," IEEE Conf. Computer Vision and Pattern Recog., 2007.

[34] M. Gupta, A. Agrawal, A. Veeraraghavan, and S. G. Narasimhan, "Structured light 3D scanning in the presence of global illumination," Proc. Conf. Computer Vision and Pattern Recog., pp. 713-720, 2011.

[35] M. O' Toole, J. Mather, and K. N. Kutulakos, "3D shape and indirect appearance by structured light transport," Proc. Conf. Computer Vision and Pattern Recog., pp. 3246-3253, 2014.

[36] N. Chiba, S. Arai, K. Hashimoto, "Feedback projection for 3D measurements under complex lighting conditions," American Control Conf., pp. 4649-4656, 2017.

[37] D. Khadraoui, G. Motyl, P. Martinet, J. Gallice, and F. Chaumette, "Visual servoing in robotics scheme using a camera/laser-stripe sensor," IEEE Trans. Robot. and Auto., vol. 12, no. 5, pp. 743-750, 1996.

[38] J. Pagès, C. Collewet, F. Chaumette, and J. Salvi, "Plane-to-plane positioning from image-based visual servoing and structured light," IEEE/RSJ Int. Conf. Intelligent Robots and Systems, pp. 1004-1009, 2004.

[39] J. Pages, C. Collewet, F. Chaumette, and J. Salvi, "Robust decoupled visual servoing based on structured light," IEEE/RSJ Int. Conf. Intelligent Robots and Systems, vol. 2, pp. 2676-2681, 2005.

[40] J. Pagès, C. Collewet, F. Chaumette, and J. Salvi, "A camera-projector system for robot positioning by visual servoing," Computer Vision and Pattern Recog. Workshop, 2006.

[41] J. Pagès, C. Collewet, F. Chaumette, and J. Salvi, "Visual Servoing by Means of Structured Light: Visual-based Robot Task Optimisation from Controlled Light Pattern Projection," 2008.

[42] V. Lippiello, B. Siciliano and L. Villani, "Position-based visual servoing in industrial multirobot cells using a hybrid camera configuration," IEEE Trans. Robot., vol. 23, no. 1, pp. 73-86, 2007.

[43] C. Collewet, and E. Marchand, "Photometric visual servoing," IEEE Trans. Robot., vol. 27, no. 4, pp.828-834, 2011.

[44] P. C Ng, and S. Henikoff, "SIFT: Predicting amino acid changes that affect protein function," Oxford University Press Nucleic acids research, vol. 31, no. 13, pp. 3812-3814, 2003.

[45] H. Bay, T. Tuytelaars, and L. V. Gool, "Surf: Speeded up robust features," Springer European conference on computer vision, pp. 404417, 2006.

[46] P. F Alcantarilla, and T. Solutions, "Fast explicit diffusion for accelerated features in nonlinear scale spaces," IEEE Transsactions on Pattern Analysis and Machine Intelligence, vol. 34, no. 7, pp. 1281-1298, 2011.

[47] G. Silveira and E. Malis,"Direct Visual Servoing: Vision-Based Estimation and Control Using Only Nonmetric Information," IEEE Transactions on Robotics, vol. 28, no. 4, pp. 974-980, 2012.

[48] A. Kaehler, and G. Bradski, "Learning OpenCV 3: computer vision in C++ with the OpenCV library," O'Reilly Media, Inc., 2016. 